\title{
POINT CLOUDS TO DIRECT INDOOR PEDESTRIAN PATHFINDING
}

\author{
J. Balado*, L. Díaz-Vilariño, P. Arias, E. Frías \\ Applied Geotechnologies Group, Dept. Natural Resources and Environmental Engineering, \\ School of Mining and Energy Engineering, University of Vigo, Campus Lagoas-Marcosende, CP 36310 Vigo, Spain \\ (jbalado, lucia, parias, efrias)@uvigo.es
}

Commission IV, WG IV/5

KEY WORDS: Physical accessibility, Obstacle detection, Graph modelling, Occlusion correction, As-built 3D

\begin{abstract}
:
Increase in building complexity can cause difficulties orienting people, especially people with reduced mobility. This work presents a methodology to enable the direct use of indoor point clouds as navigable models for pathfinding. Input point cloud is classified in horizontal and vertical elements according to inclination of each point respect to $n$ neighbour points. Points belonging to the main floor are detected by histogram application. Other floors at different heights and stairs are detected by analysing the proximity to the detected main floor. Then, point cloud regions classified as floor are rasterized to delimit navigable surface and occlusions are corrected by applying morphological operations assuming planarity and taking into account the existence of obstacles. Finally, point cloud of navigable floor is downsampled and structured in a grid. Remaining points are nodes to create navigable indoor graph. The methodology has been tested in two real case studies provided by the ISPRS benchmark on indoor modelling. A pathfinding algorithm is applied to generate routes and to verify the usability of generated graphs. Generated models and routes are coherent with selected motor skills because routes avoid obstacles and can cross areas of non-acquired data. The proposed methodology allows to use point clouds directly as navigation graphs, without an intermediate phase of generating parametric model of surfaces.
\end{abstract}

\section{INTRODUCTION}

Population increase in cities implies a building growth. Buildings are becoming more and more complex, so finding a way inside them can be a challenge. This is especially remarkable in public buildings, i.e. railway stations, airports, universities and hospitals, as well as office and residential buildings. Top views and 3D models do not always allow comfortable interpretation of buildings to search for routes. The generation of specific navigation models, such as networks and hierarchical graphs, is necessary to apply path-finding algorithms. Each model type has its own characteristics related with node locations and connections. The characteristics are reflected in the route generation (Vanclooster et al., 2016). Navigation models should include information of relevant elements and navigable space according to pedestrian needs (Park et al., 2018): entrance, rooms, stairs, elevators, etc.

In order to obtain navigation models, 3D or 2D plans of the buildings are used. But when theoretical information is nonexistent or outdated, LiDAR technology allows the acquisition of the 3D as-built environment quickly and accurately. Acquired point clouds contain geometric information and obstacle locations, not existing in the theoretical models. The disadvantage of point clouds is that they present a large amount of disordered information, density variations and occlusions. These limitations must be addressed in order to obtain complete and useful models of the scenes.

The aim of this work is to develop a methodology to use indoor point clouds directly as navigable models for pathfinding. The models generated must be coherent with user motor skills. Two different user profiles have been chosen according to two motor skills, which correspond to the displacement of persons with no reduced mobility (PnRM) and people in wheelchairs. The proposed methodology involves correcting occlusions on acquired point cloud of floor and keeping the free unobstructed space necessary for a person or a wheelchair to pass comfortably. Point cloud is firstly classified as floor, ceiling and obstacles. The point cloud corresponding to floor and obstacles is rasterized to obtain a semantically enriched image with classes. On the raster image, obstacles are expanded, according to the free unobstructed width that corresponds to each motor skill, then, floor occlusions are corrected by applying mathematical morphology operations. The last phase is devoted to uniform point density, distribute graph nodes and join them by arcs. To verify the usability of the generated graph for real route calculation, a pathfinding algorithm is applied to generate routes.

The rest of this paper is organized as follows. Section 2 collects related work about indoor modelling for path planning. Section 3 presents the designed methodology. Section 4 is devoted to analyse the results. Finally, Section 5 concludes this work.

\section{RELATED WORK}

The most common indoor environment models are navigation graphs between rooms. These models are a variation of building topological graphs. Adjacent rooms are connected through doors by arcs (Tran et al., 2017). Rooms and doors are represented as nodes located in the centroid. Different floors can also be connected via stairs or elevators (Lorenz et al., 2006). Nodes can be semantically enriched with information of type of element they represent (Fichtner et al., 2018; Ge et al.,

\footnotetext{
* Corresponding author
} 


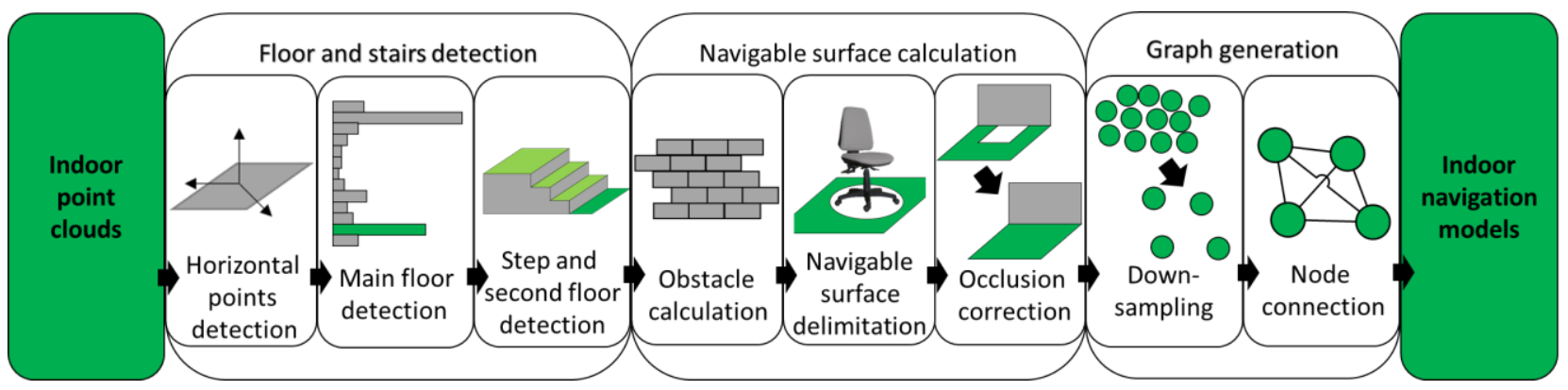

Figure 1. Workflow of the methodology

2015). Topological building networks allows an easy pathfinding between rooms and floors, but has limitations in open spaces and does not consider the existing obstacles within each room. When rooms have complex shapes, visibility analysis is used to segment them by decentralizing nodes (Stoffel et al., 2007). Another alternative is calculate new nodes in corners and new arcs, in order to generate more realistic routes (Boguslawski et al., 2016; Liu and Zlatanova, 2011; Yang and Worboys, 2015).

Topological building networks can be generated from BIM (Building Information model) (Teo and Cho, 2016) or point clouds (Jamali et al., 2017). Depending on the input data, networks can be obtained in different ways. In point clouds, doors and walls must be detected to delimit rooms (DíazVilariño et al., 2015; Nakagawa et al., 2015; Staats et al., 2018). In BIM, all elements are characterised, the application of methodologies for doors and walls detection is therefore not necessary. Sithole (2018) relates the skeleton of floor BIM elements, calculated from morphological operations, with an indoor network.

An alternative to model based on the relationship between rooms is that based on navigable space. This modelling focuses on a distribution of the existing free space inside buildings, which is discretized through the Delaunay triangulation (Krüminaitè and Zlatanova, 2014), Voronoi diagrams (Wallgrün, 2005) or octrees (Fichtner et al., 2018). In works that model space from point clouds, navigable space is influenced by floor elements existing in scenes. Staats et al. (2017) distinguish between floor and stairs, as well as dynamic objects and furniture. Fichtner et al. (2018) also distinguish between navigable surfaces (stairs and floors) and the octree that distributes space is semantically enriched and with this information. Maruyama et al., $(2017,2016)$ reduce the number of points belonging to floor in order to create a navigation graph and analyse the accessibility of the environment.

Certain applications require a more detailed modelling of the navigable environment, for example, the study of movement of crowds or evacuation in emergencies. In these cases, a cellular automata model is chosen, the floor is discretized at fixed size intervals, normally in grid or voxel-grid. The regular discretization allows a more precise location of people in the environment (Ren et al., 2017). Zhang et al. (2015) assign weights to a floor grid, related to distance to exit or dangerous zones, to calculate the movement of a crowd in an emergency situation. Ruggiero et al. (2018) employ a floor grid to study the probabilities of displacement of people. Occupation maps are another examples of cellular automata (Díaz-Vilariño et al., 2018; Xu et al., 2018), navigable pixels can be differentiated from those occupied by objects.
With regard to the mentioned works, the methodology presented in this paper exclusively employs an indoor point cloud without classifying or trajectory information as input. The floor is detected and part of its points are used as final graph nodes, distributed in the form of an 8 connected cellular automata. The rest of the elements, such as walls and furniture, are considered as obstacles. Their space and influence is eliminated from the navigable floor according to two motor skills and free unobstructed width in ISO-21.542 (ISO, 2011). Doors and other structural elements do not need to be detected and classified. Occlusions in the navigable floor are corrected.

\section{METHODOLOGY}

The input of the methodology is a point cloud of an one-plant indoor environment $\mathrm{P}(\mathrm{X}, \mathrm{Y}, \mathrm{Z})$. The methodology consists of a sequence of phases where the floor is detected, the navigable surface is delimited and the occlusions are corrected. At last, the point cloud of the navigable floor is downsampled, structured in a grid and converted into a graph. Figure 1 shows the workflow of the methodology.

\subsection{Floor and stairs detection}

To generate a precise map of the navigable surface, floors and stairs must be detected. A methodology for floor detection is developed by (Okorn et al., 2010), where floor and ceil are detected from the peaks of the $3 \mathrm{D}$ projection on vertical axes in a height histogram. The problem with this methodology is that small floors at different heights and stair segments are not detected correctly. In order to solve this, the methodology mentioned above has been modified and integrated into another major methodology explained in this sub-section.

Firstly, normals $\mathrm{N}\left(\mathrm{N}_{\mathrm{x}}, \mathrm{N}_{\mathrm{y}}, \mathrm{N}_{\mathrm{z}}\right)$ of each point with respect to its $n$ neighbours are estimated. Then, points whose normal has horizontal tilt $\mathrm{N}_{\mathrm{z}}<n t$ are separated as possible floor points $\mathrm{H}$ and others as possible obstacles $\mathrm{V}$. $\mathrm{H}$ are represented in a histogram in projection on vertical axis. The points whose bins have more weight correspond to the main floor $\mathrm{F}$ and ceiling $\mathrm{C}$, which are differentiated by height. In the remaining horizontal points $\mathrm{R}$, other fragments that also belong to the floor and stairs should be searched.

The elements Er that form $\mathrm{R}$ are individualized by connected components (Soilán et al., 2016). Point to point distances $\mathrm{D}\left(\mathrm{D}_{\mathrm{x}}, \mathrm{D}_{\mathrm{y}}, \mathrm{D}_{\mathrm{z}}\right)$ between $\mathrm{R}$ and $\mathrm{F}$ are calculated. Elements $\mathrm{Er}$ containing points with $\mathrm{D}_{\mathrm{z}}$ less than the maximum step height $s h$ and with $D_{x y}$ less than the minimum free unobstructed width fuw, are added to the floor already detected F. This process runs in a loop until new floor elements are no more detected. The 
pseudocode is indicated in Algorithm 1. This methodology ensures that false positives (i.e., chairs and tables) are not detected as floors (Figure 2). Floors more than distances sh, fuw from others are considered inaccessible.

\subsection{Navigable surface calculation}

In this phase, the navigable surface is delimited from the floor and the obstacle influence on it. The navigable floor is considered as the floor under the free unobstructed space, divided into height and width.

Firstly, the free unobstructed height condition is applied. Two height thresholds have been defined for this, a minimum height fuhl that a person can climb and a maximum height fuh2 that can pass without the danger of hitting the head (Figure 3 ). The distances between the complete floor Fc and possible obstacles ( $\mathrm{V}$ and $\mathrm{Rf}$ ) are calculated. The points with a distance between fuhl and fuh 2 thresholds are saved in an obstacle point cloud $\mathrm{O}$. Remaining points are discarded.

Secondly, the free unobstructed width condition is applied. Point clouds $\mathrm{Fc}$ and $\mathrm{O}$ are rasterized (Soilán et al., 2018). In each pixel, the mode of element class is saved: floor, obstacle or empty. The pixels corresponding to obstacles are dilated fuw, so the floor pixels in contact with them are eroded the same size.

Finally, to complete the navigable surface, holes in the point cloud of the navigable floor are filled. The occlusion correction methodology presented in (Balado et al., 2019) is used as modified. This methodology allows to complete occlusions by means of morphological operations assuming floor planarity. The modification implemented consists of generating points based on each pixel occluded, and not on polygons that group several pixels. The rasterization, application of fuw and occlusion correction are represented in Figure 4.

Algorithm 1.

Detection of steps and floors at different elevations.

Inputs: Main_Floor $\{\mathrm{F}\}$, Residual $\{\mathrm{R}\}$, step_height $s h$, free_unobstructed_width fuw

Outputs: Floor_Complete $\{\mathrm{Fc}\}$, Residual_without_Floor $\{\mathrm{Rf}\}$

Horizontal_elements $\{\mathrm{Er}\} \leftarrow$ ConnectedComponents $(\mathrm{R})$

New_Floor $\{$ Fn $\} \leftarrow F$

$\mathrm{Fc} \leftarrow \mathrm{F}$

While $\mathrm{Fn} \neq \varnothing$

Distances $\{\mathrm{D}\} \leftarrow\|\mathrm{R}, \mathrm{F}\|$

Fn $\leftarrow \mathrm{Er} \supset \mathrm{R}:(\mathrm{D}(\mathrm{xy})<$ fuw \& $\mathrm{D}(\mathrm{z})<s h)$

Er $\leftarrow$ Remove $\{$ Er $\supset \mathrm{R}:(\mathrm{D}(\mathrm{xy})<$ fuw \& $\mathrm{D}(\mathrm{z})<s h)\}$

$\mathrm{Fc} \leftarrow$ Add $\{\mathrm{Fn}\}$

End while

$\mathrm{Rf} \leftarrow \mathrm{R} \in \mathrm{Er}$

Return $\{\mathrm{Fc}, \mathrm{Rf}\}$

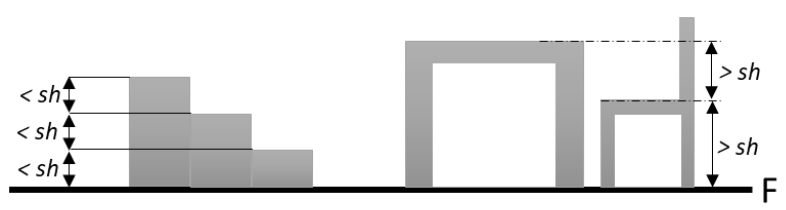

Figure 2. Comparison of distances between horizontal surfaces in relation to $s h$.

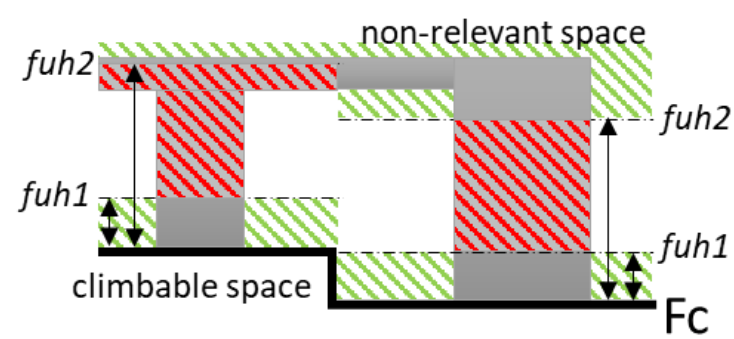

Figure 3. Relation between free unobstructed height (fuhl and fuh2) and obstacles (in red) for the navigable surface.

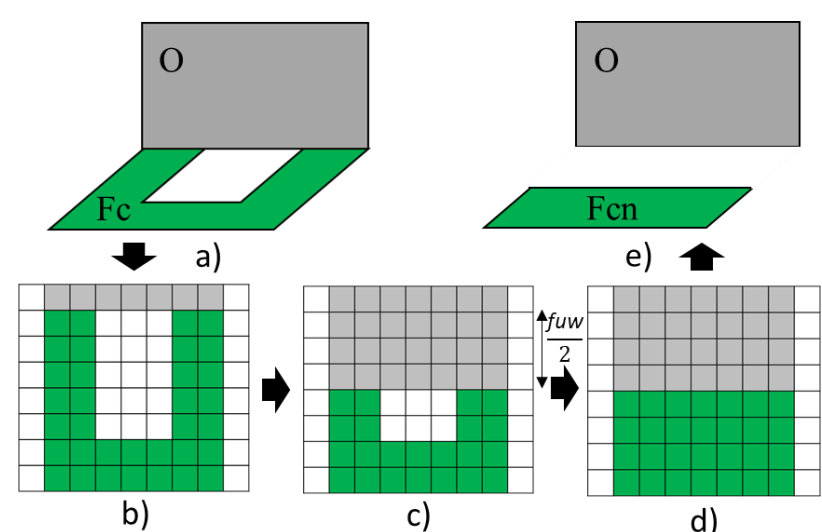

Figure 4. Process of navigable surface delimitation and occlusion correction: a) Point cloud with labeled O (gray) and

Fc (green), b) Conversion to raster, c) Dilatation fuw/2 of obstacles, d) occlusion correction, e) complete navigable floor point cloud without occlusions Fen.

\subsection{Graph generation}

At this stage of the methodology, the complete navigable floor Fen point cloud is used as nodes and in a 3D graph G. As the point cloud has great variations in point density and an excessive amount of points, a downsampled is applied to reduce and distribute the remaining points in a grid at a distance $d$ between them. These points are used as nodes in the navigation network. The points are connected to each other by searching for neighbours at distance $\mathrm{d}^{0.5}$, which ensures 8 connections of each node in different directions and more realistic routes (Bemmelen et al., 1993).

\section{RESULTS AND DISCUSSION}

\subsection{Datasets}

The methodology has been tested in two case studies provided by the ISPRS benchmark on indoor modelling (Khoshelham et al., 2017). The down floor of TUB 2 (Technische Universität Braunschweig, Germany) and Fire Brigade in Delft (The Netherlands) have been selected as case studies 1 and 2 respectively. The first one has been acquired with a Zeb-Revo sensor and presents few occlusions. The second has been acquired with Terrestrial Laser Scanner Leica C10, has occluded floor areas and numerous furniture. The methodology has been implemented in Matlab and processed on an Intel Core i7-7700HQ CPU $2.80 \mathrm{GHz}$ with $16 \mathrm{~GB}$ RAM. 


\subsection{Results of methodology application}

This sub-section aims to evaluate the quality of the graph obtained with the methodology application. In order to guarantee the reproducibility, the parameter values must be established (Table 1). Parameters $n$ and $n t$ have been calculated experimentally. Parameters sh, fuw, fuhl and fuh2 have been extracted from ISO-21-542. The parameter $d$ is related to final graph node density. In consideration of methodology is applied in indoor environments with reduced dimensions, it is estimated that $d=0.2 \mathrm{~m}$ is a good balance between route precision and number of nodes in the final graph.

The quality of the final indoor navigation model depends on the intermediate results of the methodology phases. The correct detection of floor and stairs, the obstacle detection and occlusion correction allow to delimit the whole navigable surface. Obstacles are represented on the navigable surface as areas without navigation nodes. The two thresholds fuhl and fuh2 differentiate obstacles for PnRM and wheelchairs, for example, in areas with stairs (Figure 5). The methodology application also considers people and closed doors as obstacles. These situations produce the disappearance of floor navigable areas and cause breaks in the continuity of the generated models. Therefore, during the acquisition process it is recommended that doors are open and there are no people in the area.

Most of navigable surface in occluded areas has been regenerated. Non-regenerated areas correspond to locations on the point cloud border with incomplete walls, because occlusions are confused with the building outside (Figure 6).

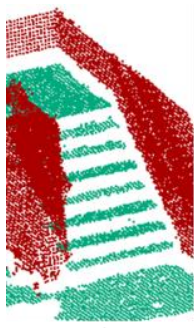

a)

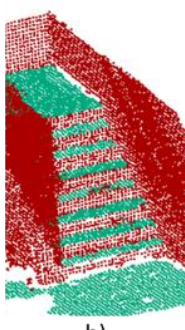

b)
Figure 5. Stairs in case study 1. Obstacles (in red) considered for PnRM (a) and for wheelchairs (b) on the floor and stairs (in green).

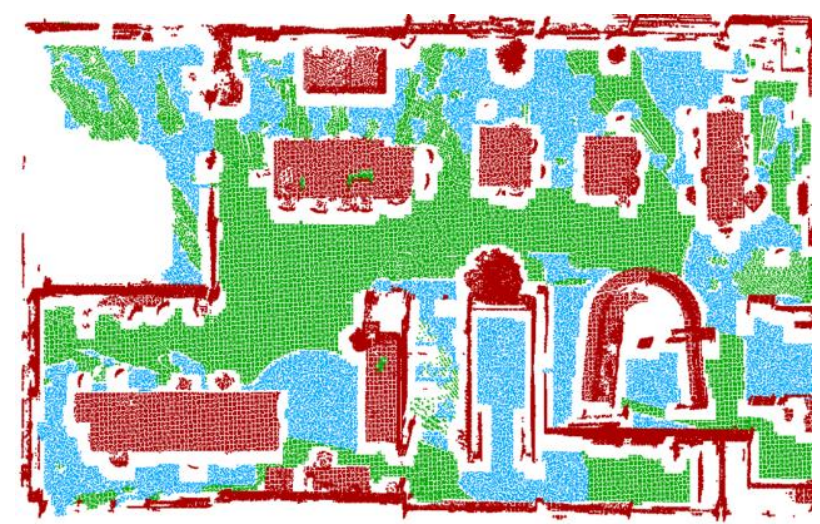

Figure 6. Top view of case study 2 after occlusion correction. Colour code: Obstacles in red, floor occluded corrected in blue, remaining navigable floor in green.
Navigable surface has been correctly delimited according to free unobstructed space left by obstacles, different for each motor skill (Figure 7). Final navigation nodes have been distributed uniformly on the navigable surface by the downsampled and the structuring (Figure 8). No obstacle has been omitted. The number of nodes needed to create a detailed interior navigation model was $0.01 \%$ of the number of points in the initial cloud (Table 2).

\begin{tabular}{lccc}
\hline \multicolumn{1}{c}{ Parameter } & Abbrev. & $\begin{array}{c}\text { Value for } \\
\text { PnRM }\end{array}$ & $\begin{array}{c}\text { Value for } \\
\text { wheelchairs }\end{array}$ \\
\hline $\begin{array}{l}\text { Number of } \\
\text { neighbours }\end{array}$ & $n$ & \multicolumn{2}{c}{10 points } \\
\hline $\begin{array}{l}\text { Normal threshold for } \\
\text { horizontality }\end{array}$ & $n t$ & \multicolumn{2}{c}{0.9} \\
\hline Step height & $s h$ & \multicolumn{2}{c}{$0.15 \mathrm{~m}$} \\
\hline $\begin{array}{l}\text { Free unobstructed } \\
\text { width }\end{array}$ & $f u w$ & $0.8 \mathrm{~m}$ & $1 \mathrm{~m}$ \\
\hline $\begin{array}{l}\text { Free unobstructed } \\
\text { height 1 }\end{array}$ & $f u h 1$ & $0.15 \mathrm{~m}$ & $0 \mathrm{~m}$ \\
\hline $\begin{array}{l}\text { Free unobstructed } \\
\text { height 2 }\end{array}$ & $f u h 2$ & $2 \mathrm{~m}$ & $2 \mathrm{~m}$ \\
\hline Node distance & $d$ & $0.5 \mathrm{~m}$ & $0.5 \mathrm{~m}$ \\
\hline
\end{tabular}

Table 1. Parameter values.

\begin{tabular}{|c|c|c|c|c|}
\hline Dataset & $\begin{array}{c}\text { Initial } \\
\text { point } \\
\text { number }\end{array}$ & $\begin{array}{l}\text { Motor } \\
\text { skill }\end{array}$ & $\begin{array}{c}\text { Remaining } \\
\text { node } \\
\text { number }\end{array}$ & $\begin{array}{c}\text { Processing } \\
\text { time }\end{array}$ \\
\hline \multirow{2}{*}{1} & \multirow{2}{*}{$13.1 \mathrm{M}$} & $\mathrm{p}$ & 979 & $11 \mathrm{~m} \mathrm{42s}$ \\
\hline & & W & 865 & $11 \mathrm{~m} \mathrm{50s}$ \\
\hline \multirow{2}{*}{2} & \multirow{2}{*}{$7.3 \mathrm{M}$} & $\mathrm{p}$ & 1918 & $10 \mathrm{~m} 23 \mathrm{~s}$ \\
\hline & & $\mathrm{w}$ & 1566 & $10 \mathrm{~m} \mathrm{57s}$ \\
\hline
\end{tabular}

Table 2. Point cloud size and time processing.

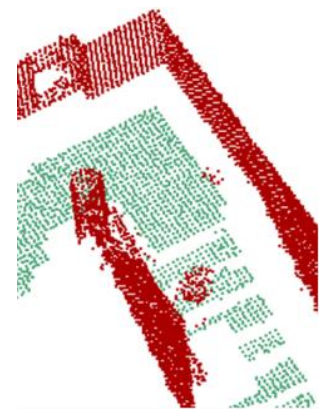

a)

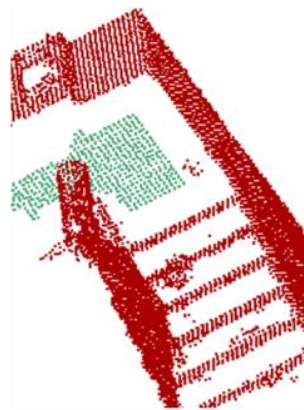

b)
Figure 7. Navigable floor (green) of stairs in case study 1 for PnRM (a) and for wheelchairs (b). Obstacles in red.

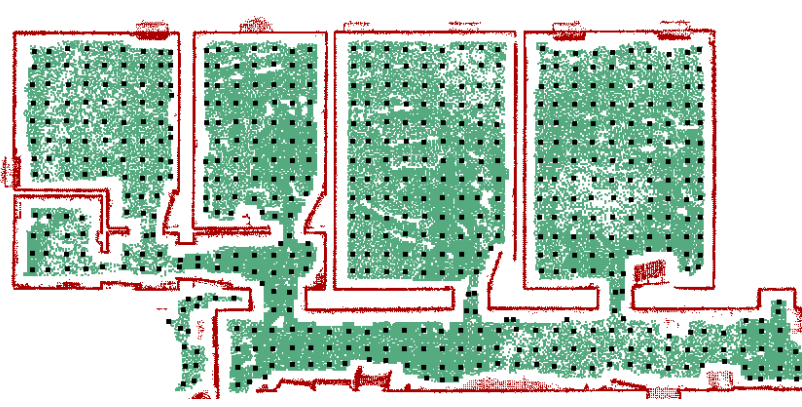

Figure 8. Node distribution in case study 1 for PnRM. Colour code: nodes in black, obstacles in red and navigable floor in green. 


\subsection{Results of pathfinding application}

To test if the generated models are suitable for pedestrian pathfinding, Dijkstra algorithm has been implemented to calculate routes between two nodes. Based on knowledge about the indoor environment of both case studies, it has been proved visually that the generated routes avoid obstacles and can generate routes between and inside rooms (Figure 9 and Figure $10)$.

Due to floor and stairs detection and the consideration of different obstacles for each motor skill, routes generated for PnRM profiles can climb stairs (Figure 11). In the case of wheelchair profiles, the stairs are not passable no routes can be generated over them. The occlusion correction has been allowed to generate routes through areas not acquired due to occlusions in the input point cloud (Figure 12). The higher processing time of pathfinding has not exceeded 0.02 seconds to calculate a route 24 metres long. The generated models would allow the application of pathfinding algorithms in real time.

\section{CONCLUSIONS}

In this work, a methodology to enable the direct use of indoor point clouds as navigable models for pathfinding is presented. The models generated are more detailed than other alternatives based on topological relationships between rooms. The methodology uses point clouds as input data only with geometric information, not semantic as in BIM.

The designed methodology allows to obtain a model delimited by the existing obstacles and the free unobstructed space needed by each user. Occlusions in point cloud of the navigable floor have been corrected. Thus, it is not necessary to use complete acquired areas, which is difficult due to furniture existing in indoors. Direct use of points as final nodes allows the generation of routes without an intermediate phase of generating parametric model of surfaces.

Routes generated in case study models have been shown to be navigable for both motor skills, PnRM and wheelchairs. In addition, the processing time of the route calculation makes the proposed models suitable for real time application.

Future work will focus on adapting the methodology to work on different building floors at the same time. The application of the methodology to new case studies with a greater complexity in floor elements for PnRM and wheelchairs will also be valued.

\section{ACKNOWLEDGEMENTS}

Authors would like to thank to the Universidade de Vigo for the financial support (00VI 131H 641.02), the Xunta de Galicia given through human resources grant (ED481B 2016/079-0) and competitive reference groups (ED431C 2016-038), and the Ministerio de Economia, Industria y Competitividad -Gobierno de España- (TIN2016-77158-C4-2-R, RTC-2016-5257-7). This project has received funding from the European Union's Horizon 2020 research and innovation programme under grant agreement No 769255. This document reflects only the author's view and the Agency is not responsible for any use that may be made of the information it contains. The statements made herein are solely the responsibility of the authors.

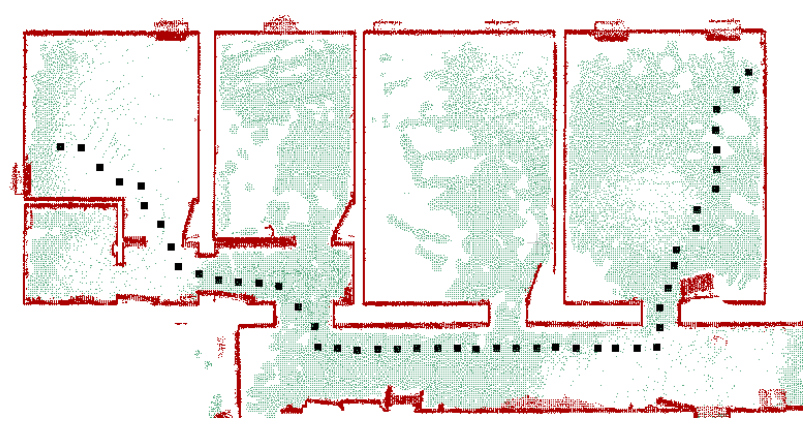

Figure 9. Route between rooms in case study 1. Colour code: acquired floor in green, obstacles in red and route nodes in black.

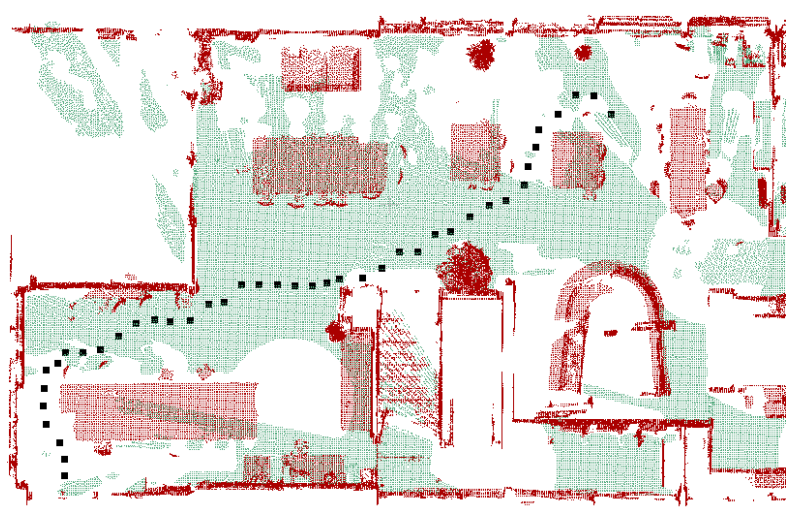

Figure 10. Route inside room in case study 2. Colour code: acquired floor in green, obstacles in red and route nodes in black.

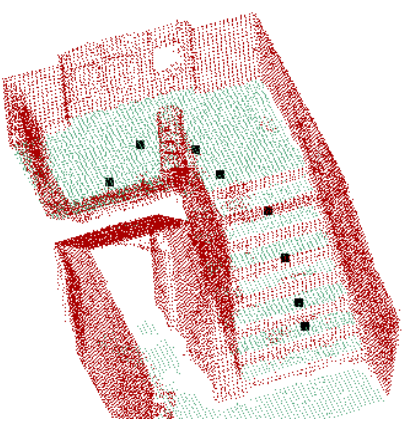

Figure 11. Route in stairs for PnRM in case study 1. Colour code: acquired floor in green, obstacles in red and route nodes in black.

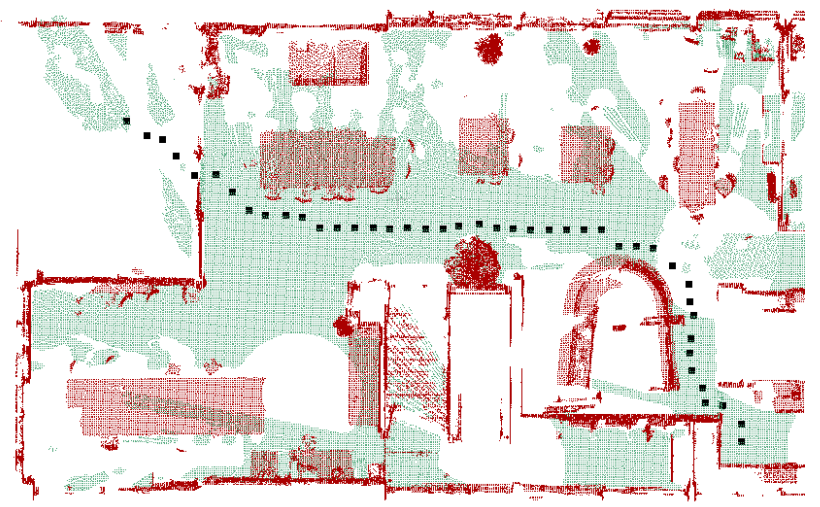

Figure 12. Route over occluded areas in case study 2. Colour code: acquired floor in green, obstacles in red and route nodes in black. 


\section{REFERENCES}

Balado, J., Díaz-Vilariño, L., Arias, P., Lorenzo, H., 2019. Point clouds for direct pedestrian pathfinding in urban environments. ISPRS J. Photogramm. Remote Sens. 148, 184-196. https://doi.org/10.1016/j.isprsjprs.2019.01.004

Bemmelen, J. Van, Quak, W., Hekken, M. Van, Oosterom, P. Van, 1993. Vector vs. Raster-based Algorithms for Cross Country Movement Planning. Proc. AutoCarto 11, 304-317.

Boguslawski, P., Mahdjoubi, L., Zverovich, V., Fadli, F., 2016. Two-Graph Building Interior Representation for Emergency Response Applications. ISPRS Ann. Photogramm. Remote Sens. Spat. Inf. Sci. III-2, 9-14. https://doi.org/10.5194/isprsannals-III-2-9-2016

Díaz-Vilariño, L., Frías, E., Balado, J., González-Jorge, H., 2018. Sanc planning and route optimization for control of execution of as-designed BIM. ISPRS - Int. Arch. Photogramm. Remote Sens. Spat. Inf. Sci. XLII-4, 143-148. https://doi.org/10.5194/isprs-archives-XLII-4143-2018

Díaz-Vilariño, L., Khoshelham, K., Martínez-sánchez, J., Arias, P., 2015. 3D Modeling of Building Indoor Spaces and Closed Doors from Imagery and Point Clouds. Sensors 15, 3491-3512. https://doi.org/10.3390/s150203491

Fichtner, F.W., Diakité, A.A., Zlatanova, S., Voûte, R., 2018. Semantic enrichment of octree structured point clouds for multi-story 3D pathfinding. Trans. GIS 22, 233-248. https://doi.org/10.1111/tgis.12308

Ge, H., Bessho, M., Koshizuka, N., Sakamura, K., 2015. Model Design of Generating Path with Accessibility Semantics for Assisting Indoor Mobility, in: 2015 IEEE International Conference on Data Science and Data Intensive Systems (DSDIS). pp. 139-146. https://doi.org/10.1109/DSDIS.2015.55

ISO, 2011. ISO-21542 Building construction Accessibility and usability of the built environment. ISO Int. Organ. Stand.

Jamali, A., Abdul Rahman, A., Boguslawski, P., Kumar, P., Gold, C.M., 2017. An automated 3D modeling of topological indoor navigation network. GeoJournal 82, 157-170. https://doi.org/10.1007/s10708-015-9675-x

Khoshelham, K., Díaz Vilariño, L., Peter, M., Kang, Z., Acharya, D., 2017. The ISPRS benchmark on indoor modelling. ISPRS - Int. Arch. Photogramm. Remote Sens. Spat. Inf. Sci. XLII-2/W7, 367-372. https://doi.org/10.5194/isprs-archives-XLII-2-W7-3672017

Krüminaitè, M., Zlatanova, S., 2014. Indoor Space Subdivision for Indoor Navigation, in: Proceedings of the Sixth ACM SIGSPATIAL International Workshop on Indoor Spatial Awareness, ISA '14. ACM, New York, NY, USA, pp. 25-31. https://doi.org/10.1145/2676528.2676529

Liu, L., Zlatanova, S., 2011. A "Door-To-Door" PathFinding Approach For Indoor Navigation. Proc. Gi4DM 2011 Geoinf. Disaster Manag. Antalya, Turkey, 3-8 May
$20113-8$.

Lorenz, B., Ohlbach, H.J., Stoffel, E.-P., 2006. A Hybrid Spatial Model for Representing Indoor Environments, in: Proceedings of the 6th International Conference on Web and Wireless Geographical Information Systems, W2GIS'06. Springer-Verlag, Berlin, Heidelberg, pp. 102-112. https://doi.org/10.1007/11935148_10

Maruyama, T., Kanai, S., Date, H., Tada, M., 2017. Simulation-Based Evaluation of Ease of Wayfinding Using Digital Human and As-Is Environment Models. ISPRS Int. J. Geo-Information https://doi.org/10.3390/ijgi6090267

Maruyama, T., Kanai, S., Date, H., Tada, M., 2016. Motion-capture-based walking simulation of digital human adapted to laser-scanned 3D as-is environments for accessibility evaluation. J. Comput. Des. Eng. 3, 250 265.

https://doi.org/https://doi.org/10.1016/j.jcde.2016.03.001

Nakagawa, M., Yamamoto, T., Tanaka, S., Shiozaki, M., Ohhashi, T., 2015. Topological 3D modeling using indoor mobile lidar data. ISPRS - Int. Arch. Photogramm. Remote Sens. Spat. Inf. Sci. XL-4/W5, 1318. https://doi.org/10.5194/isprsarchives-XL-4-W5-132015

Okorn, B., Xiong, X., Akinci, B., 2010. Toward Automated Modeling of Floor Plans, in: Fifth International Symposium on 3D Data Processing, Visualization \& Transmission. Paris, France.

Park, S., Kim, S., Yu, K., 2018. Designing of Indoor Linkable Pedestrian Network Data Model for the Transportation Vulnerable, in: Proceedings of the $2 \mathrm{Nd}$ International Conference on Digital Signal Processing, ICDSP 2018. ACM, New York, NY, USA, pp. 57-60. https://doi.org/10.1145/3193025.3193048

Ren, M., Guo, H., Shi, J., Meng, J., 2017. Indoor Pedestrian Navigation Based on Conditional Random Field Algorithm. Micromachines 8. https://doi.org/10.3390/mi8110320

Ruggiero, L., Charitha, D., Xiang, S., Lucia, B., 2018. Investigating pedestrian navigation in indoor open space environments using big data. Appl. Math. Model. 62, 499-509.

https://doi.org/https://doi.org/10.1016/j.apm.2018.06.014

Sithole, G., 2018. Indoor space routing graphs: visibility, encoding, encryption and attenuation. ISPRS - Int. Arch. Photogramm. Remote Sens. Spat. Inf. Sci. XLII-4, 579 585. https://doi.org/10.5194/isprs-archives-XLII-4-5792018

Soilán, M., Riveiro, B., Martínez-Sánchez, J., Arias, P., 2016. Segmentation and classification of road markings using MLS data. ISPRS J. Photogramm. Remote Sens. 123 , 94-103. https://doi.org/10.1016/j.isprsjprs.2016.11.011

Soilán, M., Truong-Hong, L., Riveiro, B., Laefer, D., 2018. Automatic extraction of road features in urban environments using dense ALS data. Int. J. Appl. Earth Obs. Geoinf. 64, 226-236. https://doi.org/https://doi.org/10.1016/j.jag.2017.09.010

Staats, B.R., Diakité, A.A., Voûte, R.L., Zlatanova, S., 
2018. Detection of doors in a voxel model, derived from a point cloud and its scanner trajectory, to improve the segmentation of the walkable space. Int. J. Urban Sci. 122. https://doi.org/10.1080/12265934.2018.1553685

Staats, B.R., Diakité, A.A., Voûte, R.L., Zlatanova, S., 2017. Automatic generation of indoor navigable space using a point cloud and its scanner trajectory. ISPRS Ann. Photogramm. Remote Sens. Spat. Inf. Sci. 4, 393400. https://doi.org/10.5194/isprs-annals-IV-2-W4-3932017

Stoffel, E.-P., Lorenz, B., Ohlbach, H.J., 2007. Towards a Semantic Spatial Model for Pedestrian Indoor Navigation, in: Hainaut, J.-L., Rundensteiner, E.A., Kirchberg, M., Bertolotto, M., Brochhausen, M., Chen, Y.-P.P., Cherfi, S.S.-S., Doerr, M., Han, H., Hartmann, S., Parsons, J., Poels, G., Rolland, C., Trujillo, J., Yu, E., Zimányie, E. (Eds.), Advances in Conceptual Modeling - Foundations and Applications. Springer Berlin Heidelberg, Berlin, Heidelberg, pp. 328-337.

Teo, T.-A., Cho, K.-H., 2016. BIM-oriented indoor network model for indoor and outdoor combined route planning. Adv. Eng. Informatics 30, 268-282. https://doi.org/https://doi.org/10.1016/j.aei.2016.04.007

Tran, H., Khoshelham, K., Kealy, A., D।'liaz-Vilariño, L., 2017. Extracting topological relation between indoor spaces from point clouds. ISPRS Ann. Photogramm. Remote Sens. Spat. Inf. Sci. IV-2/W4, 401-406. https://doi.org/10.5194/isprs-annals-IV-2-W4-401-2017

Vanclooster, A., Van de Weghe, N., De Maeyer, P., 2016. Integrating Indoor and Outdoor Spaces for Pedestrian Navigation Guidance: A Review. Trans. GIS 20, 491-525. https://doi.org/10.1111/tgis.12178

Wallgrün, J.O., 2005. Autonomous Construction of Hierarchical Voronoi-Based Route Graph Representations, in: Freksa, C., Knauff, M., KriegBrückner, B., Nebel, B., Barkowsky, T. (Eds.), Spatial Cognition IV. Reasoning, Action, Interaction. Springer Berlin Heidelberg, Berlin, Heidelberg, pp. 413-433.

Xu, W., Liu, L., Zlatanova, S., Penard, W., Xiong, Q., 2018. A pedestrian tracking algorithm using grid-based indoor model. Autom. Constr. 92, 173-187. https://doi.org/https://doi.org/10.1016/j.autcon.2018.03.0 31

Yang, L., Worboys, M., 2015. Generation of navigation graphs for indoor space. Int. J. Geogr. Inf. Sci. 29, 17371756. https://doi.org/10.1080/13658816.2015.1041141

Zhang, W., Zhou, Y., Tian, W., Hu, B., 2015. GIS-based indoor pedestrian evacuation simulation combining the particle swarm optimization and the floor field model, in: 2015 23rd International Conference on Geoinformatics. pp. 1-4 https://doi.org/10.1109/GEOINFORMATICS.2015.7378 551 